\title{
On the realization of the bulk modulus bounds for two-phase viscoelastic composites
}

\author{
Andreasen, Casper Schousboe; Andreassen, Erik; Jensen, Jakob Søndergaard; Sigmund, Ole
}

Published in:

Journal of the Mechanics and Physics of Solids

Link to article, DOI:

10.1016/j.jmps.2013.09.007

Publication date:

2014

Link back to DTU Orbit

Citation (APA):

Andreasen, C. S., Andreassen, E., Jensen, J. S., \& Sigmund, O. (2014). On the realization of the bulk modulus bounds for two-phase viscoelastic composites. Journal of the Mechanics and Physics of Solids, 63, 228-241. https://doi.org/10.1016/j.jmps.2013.09.007

\section{General rights}

Copyright and moral rights for the publications made accessible in the public portal are retained by the authors and/or other copyright owners and it is a condition of accessing publications that users recognise and abide by the legal requirements associated with these rights.

- Users may download and print one copy of any publication from the public portal for the purpose of private study or research.

- You may not further distribute the material or use it for any profit-making activity or commercial gain

- You may freely distribute the URL identifying the publication in the public portal 


\title{
On the realization of the bulk modulus bounds for two-phase viscoelastic composites
}

\author{
Casper Schousboe Andreasen, Erik Andreassen, Jakob Søndergaard Jensen, \\ Ole Sigmund \\ Department of Mechanical Engineering, Section for Solid Mechanics, Technical \\ University of Denmark, Nils Koppels Allé, Building 404, DK-2800 Kgs. Lyngby
}

\begin{abstract}
Materials with good vibration damping properties and high stiffness are of great industrial interest. In this paper the bounds for viscoelastic composites are investigated and material microstructures that realize the upper bound are obtained by topology optimization. These viscoelastic composites can be realized by additive manufacturing technologies followed by an infiltration process. Viscoelastic composites consisting of a relatively stiff elastic phase, e.g. steel, and a relatively lossy viscoelastic phase, e.g. silicone rubber, have non-connected stiff regions when optimized for maximum damping. In order to ensure manufacturability of such composites the connectivity of the matrix is ensured by imposing a conductivity constraint and the influence on the bounds is discussed.
\end{abstract}

Keywords: viscoelasticity, topology optimization, microstructure, analytical bounds

\section{Introduction}

Materials with enhanced vibration damping capabilities are of great interest in a variety of industrial and personal appliances ranging from pumps over satellite communication equipment to phono cartridges. Typical structural materials such as steel display low intrinsic damping and additional

Email address: csan@mek.dtu.dk (Casper Schousboe Andreasen)

Preprint submitted to Journal of the Mechanics and Physics of Solids January 9, 2014 
measures need to be taken to damp unwanted vibrations. A composite material such as gray cast iron shows significant damping in the order of 1-1.5\% of the critical damping due to its graphite content (Millet et al., 1981), however, metallic materials in general exhibit damping less than $1 \%$. Cast iron is just one composite material to consider for increased damping, other cast composites could potentially have larger damping ratios. The development of viscoelastic composites has attained quite some attention in order to develop materials with both high stiffness and high damping (Brodt and Lakes, 1995).

One production method for generating advanced composites with architectured microstructure is additive manufacturing (AM), e.g. selective laser sintering/melting where it is possible to manufacture composite materials with a geometrically advanced metallic matrix which afterwards can be infiltrated by a viscoelastic material. These advanced composites may exhibit very large damping, though obviously, limited by the theoretical bounds.

For low frequency harmonic vibrations where the inertia effects can be neglected the loss can be described by the elasticity equations using a complex modulus of elasticity (Hashin, 1970) according to the correspondence principle (Hashin, 1965). The theoretical bounds for the complex stiffness of viscoelastic composites have been developed with offset in the corresponding bounds for elastic composites by Hashin and Shtrikman (1963). The bounds for the complex material properties are derived using the translation method developed by Cherkaev and Gibiansky (1994). In Gibiansky and Milton (1993), and Gibiansky and Lakes (1993) the bounds for the complex bulk modulus for two- and three-dimensional two-phase isotropic viscoelastic composites are derived for a fixed and arbitrary volume fraction, respectively. Gibiansky and Lakes (1997) summarize the bounds, both for complex bulk and shear moduli of two-dimensional two-phase isotropic viscoelastic composites and present a method for computing the bounds. The bounds for the three-dimensional two-phase complex shear modulus are presented in Milton and Berryman (1997).

The structures that realize the viscoelastic bounds include multiscale structures such as the Hashin coated spheres assemblage (Hashin and Shtrikman, 1963) and rank-N laminates (Lurie and Cherkaev, 1986; Francfort and Murat, 1986). These structures are also optimal for elastic materials with respect to stiffness. For elastic materials the bounds have been realized using single scale structures in Vigdergauz (1994) and two scale structures in Sigmund (2000) which presumably also apply to the viscoelastic bounds as 
it does for the coated spheres assemblage.

The structure of viscoelastic composites has been studied by e.g. Yi et al. (1998) where the effective viscoelastic stiffness for a certain composite is computed by homogenization. The approach is extended in Yi et al. (2000) where viscoelastic composites are optimized using topology optimization for increased damping using an inverse homogenization approach similar to the one introduced in Sigmund $(1994,1995)$ for elastic materials. The inverse homogenization approach has also been used in e.g. Otomori et al. (2012) in order to obtain materials with prescribed electromagnetic permittivity for studying the bounds for this complex-valued material property.

The inverse homogenization concept is taken even further in this paper where the microstructures of the viscoelastic composites that realize the theoretical bounds, especially the upper bound, for the viscoelastic bulk modulus are assessed. An important contribution is the development of numerical bounds due to the influence of a connectivity constraint. This is an important bound as it applies to manufacturing processes, such as additive manufacturing, where the metallic phase needs to be connected.

The paper is organized as follows. The viscoelastic model is introduced in Section 2 along with the corresponding bounds. Section 3 presents the homogenization procedure and the topology optimization problem to be solved. The implementation is described in Section 4 and the results are presented in Section 5. Finally, the paper is concluded in Section 6.

\section{Method and Materials}

In this paper a linear viscoelastic material is modeled which is governed by the conservation of momentum, here in time-harmonic form

$$
\nabla_{X} \cdot \boldsymbol{\sigma}+\rho \omega^{2} \mathbf{u}=0
$$

where the capital $X$ denotes the dimensional coordinates, $\boldsymbol{\sigma}$ is the stress tensor which for viscoelastic materials is frequency dependent, $\rho$ is the mass density, $\omega$ the frequency, and $\mathbf{u}$ the displacement vector.

The stress-strain relation, Hooke's law, for an isotropic material may be written as a combination of the volumetric and deviatoric contributions as

$$
\sigma_{i j}(\omega)=\frac{E(\omega)}{d(1+\nu(1-d))} \varepsilon_{k k} \delta_{i j}+\frac{E(\omega)}{1+\nu}\left(\varepsilon_{i j}-\frac{1}{d} \delta_{i j} \varepsilon_{k k}\right)
$$


where $E(\omega)$ is the frequency-dependent stiffness at frequency $\omega$ and $\nu$ is the Poisson's ratio. The parameter $d=\{2,3\}$ is the spatial dimension and $\varepsilon_{i j}$ is the infinitesimal strain defined as

$$
\varepsilon_{i j}(\mathbf{u})=\frac{1}{2}\left(\frac{\partial u_{i}}{\partial X_{j}}+\frac{\partial u_{j}}{\partial X_{i}}\right)
$$

The stiffness of the viscoelastic material is modeled by the Standard Linear Solid (SLS) model (Zener, 1948) which is a relatively simple three parameter model for viscoelastic materials. A spring is assembled in series with a dashpot, a so-called Maxwell element, while another spring is parallel to this assembly. The SLS model is a further development of the Maxwell model, which suffers from vanishing stiffness at infinity. This is addressed by the introduction of the additional spring. The derivation is considered in Appendix A. For a harmonic excitation, the stiffness and the damping can be represented by a complex frequency-dependent stiffness

$$
k^{*}(\omega)=k^{\prime}+i k^{\prime \prime}=k_{0}+\frac{k_{m} \omega^{2} \tau^{2}}{1+\omega^{2} \tau^{2}}+i \frac{k_{m} \omega \tau}{1+\omega^{2} \tau^{2}}
$$

where $\tau=\mu / k_{m}$ is a characteristic relaxation time i.e. the ratio of viscosity $\mu$ and stiffness of the spring $k_{m}$ in series with the dashpot and $k_{0}$ is the stiffness of the spring assembled in parallel. The asterisk indicates a complex number and the single prime denotes the real part of the modulus, which is referred to as the storage modulus, while the double prime denotes the complex part, which is referred to as the loss modulus. This one-dimensional model is used to model the stiffness of the continuum, E, while assuming that the Poisson's ratio is constant, i.e. independent of frequency, and equal to $\nu=0.3$. The physical consequence of this is the neglection of the possible phase lag between the longitudinal and transversal strains (common relaxation function for both bulk and shear modulus) which may have an impact on the damping properties of the composites (Meaud and Hulbert, 2012). Furthermore Poisson's ratio will be constant in time which is debatable if e.g. a polymer is used as a constituent cf. Lakes and Wineman (2006). The restriction to the choice of materials is only chosen to limit the amount of parameters that change with frequency. The presented procedure for obtaining bounds is general and works independently of these parameter choices.

In order to generalize the approach the governing equations (1)-(4) are non-dimensionalized. Since the effective viscoelastic properties of the twomaterial composites will be considered, the non-dimensionalization is based 
on the properties of a reference material, this could e.g. be the softest material. The following relations are introduced with $\widetilde{\square}$ denoting the nondimensionalized quantities

$$
\widetilde{E}^{*}=\frac{E^{*}}{E_{0}^{r e f}}, \quad \widetilde{x}_{i}=X_{i} / L_{0}, \quad \widetilde{u}_{i}=u_{i} / L_{0}, \quad \widetilde{\rho}=\rho / \rho_{0}^{r e f}, \quad \widetilde{\omega}=\sqrt{\frac{\rho_{0}^{r e f}}{E_{0}^{r e f}}} L_{0} \omega
$$

where $E_{0}^{r e f}$ is the stiffness of the reference material at zero frequency, $L_{0}$ the characteristic length e.g. the unit cell size and $\rho_{0}^{r e f}$ the mass density of the reference material. This yields the following non-dimensional equations

$$
\begin{aligned}
& \nabla_{\widetilde{x}} \cdot \widetilde{\boldsymbol{\sigma}}+\widetilde{\rho \omega} \widetilde{\omega}^{2} \widetilde{\mathbf{u}}=0 \\
& \widetilde{\sigma}_{i j}=\frac{\widetilde{E}^{*}}{d(1+\nu(1-d))} \widetilde{\varepsilon}_{k k} \delta_{i j}+\frac{\widetilde{E}^{*}}{1+\nu}\left(\widetilde{\varepsilon}_{i j}-\frac{1}{d} \delta_{i j} \widetilde{\varepsilon}_{k k}\right) \\
& \widetilde{\varepsilon}_{i j}(\widetilde{\mathbf{u}})=\frac{1}{2}\left(\frac{\partial \widetilde{u}_{i}}{\partial \widetilde{x}_{j}}+\frac{\partial \widetilde{u}_{j}}{\partial \widetilde{x}_{i}}\right) \\
& \widetilde{E}^{*}=\widetilde{E}_{0}+\frac{\widetilde{E}_{m} \widetilde{\omega}^{2} \widetilde{\tau}^{2}}{1+\widetilde{\omega}^{2} \widetilde{\tau}^{2}}+i \frac{\widetilde{E}_{m} \widetilde{\omega} \widetilde{\tau}}{1+\widetilde{\omega}^{2} \widetilde{\tau}^{2}}
\end{aligned}
$$

where

$$
\widetilde{E}_{0}=E_{0} / E_{0}^{r e f}, \quad \widetilde{E}_{m}=E_{m} / E_{0}^{r e f}, \quad \widetilde{\tau}=\sqrt{\frac{E_{0}^{r e f}}{\rho_{0}^{r e f}}} \frac{\tau}{L_{0}}
$$

This paper is focused on the damping of vibrations and thus it is appropriate to introduce the loss factor

$$
\eta=2 \xi=\tan \delta=\frac{\widetilde{E}^{\prime \prime}}{\widetilde{E}^{\prime}}
$$

where $\xi$ is the damping ratio, and $\delta$ the phase angle between cyclic stress and strain.

Furthermore, the vibrations considered are assumed to be in the low frequency range such that quasi-static conditions apply, i.e. the forces due to inertia are negligible and the inertial term in (5) can be neglected.

For the optimization results presented, two base materials with different time-dependent behavior are used. The material parameters used are given in terms of stiffnesses and relaxation times for each of the two materials and 
are presented in Table 1. One is acting almost as an elastic material i.e. having a large long-term storage modulus, while the other is more rubbery with a large instantaneous modulus and a lower long-term storage modulus.

\begin{tabular}{l|l|l|l|l|l} 
& $\widetilde{E}_{0}$ & $\widetilde{E}_{m}$ & $\widetilde{\tau}$ & $\widetilde{E}^{\prime}$ at $\widetilde{\omega}=0.01$ & $\widetilde{E}^{\prime \prime}$ at $\widetilde{\omega}=0.01$ \\
\hline Material 1 & $20-10^{-5}$ & $10^{-5}$ & $10^{7}$ & 20 & $10^{-10}$ \\
Material 2 & 3 & 17 & 5.882 & 3.059 & 0.997
\end{tabular}

Table 1: Non-dimensional material parameters for viscoelastic base materials.

Using the above mentioned material model the stiffness depends on the frequency $\widetilde{\omega}$ as seen from (8) and this is illustrated in Fig. 1 where the storage and loss moduli are plotted versus frequency. The frequency dependence of the viscoelastic material is most pronounced in the two decades from $\widetilde{\omega}=0.01$ to $\widetilde{\omega}=1$ in which the storage modulus increases and the damping peaks. The almost elastic material (number 1) has vanishing frequency-dependence. For low frequencies the viscoelastic material (number 2) is relatively soft while it hardens for higher frequencies. In the transition zone from soft to hard the loss modulus is increasing, which is also reflected in Fig. 2 where the loss tangent for both materials is plotted versus the frequency.

The material bounds for isotropic viscoelastic materials are usually not stated using the stiffness, $E$, and Poisson's ratio, $\nu$, but generally in terms of bulk, $K=\frac{E}{3(1+2 \nu)}$ and shear $G=\frac{E}{2(1+\nu)}$ modulus. Furthermore, in order to simplify the notation, the tilde will be omitted in the following sections and the quantities are thus the non-dimensionalized versions.

\subsection{Bounds for viscoelastic composites}

The bounds for composites of viscoelastic materials have been investigated in the papers by Gibiansky and Milton (1993); Gibiansky and Lakes (1993, 1997); Milton and Berryman (1997) among others. In comparison to the bounds for elastic materials where the bounds for a given volume ratio of the constituents will be given by two numbers, the bounds for the viscoelastic moduli enclose an area in the complex plane. The frequency dependence of the bounds for the moduli of a composite consisting of the aforementioned constituents are illustrated in Fig. 3 for both the bulk and shear modulus.

The bounds are lens shaped with end points that correspond to the material properties of each phase. When the frequency is increased, the moduli 


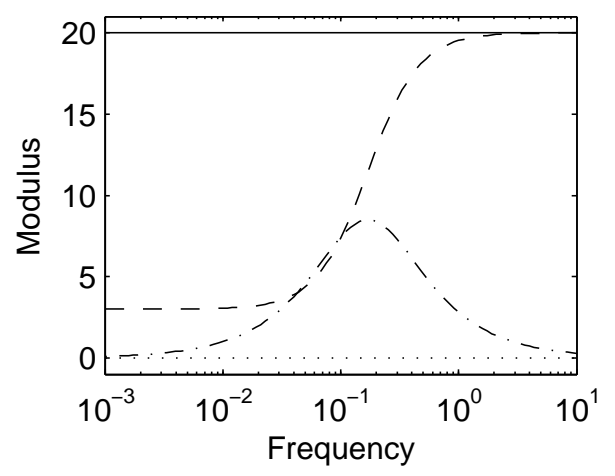

Figure 1: Frequency dependence of the storage modulus $\widetilde{E}^{\prime}$ and the loss modulus $\widetilde{E}^{\prime \prime}$. The solid and dotted lines denote the storage and loss modulus of material 1 , respectively. The dashed and dash-dot lines denote the storage and loss modulus of material 2, respectively.

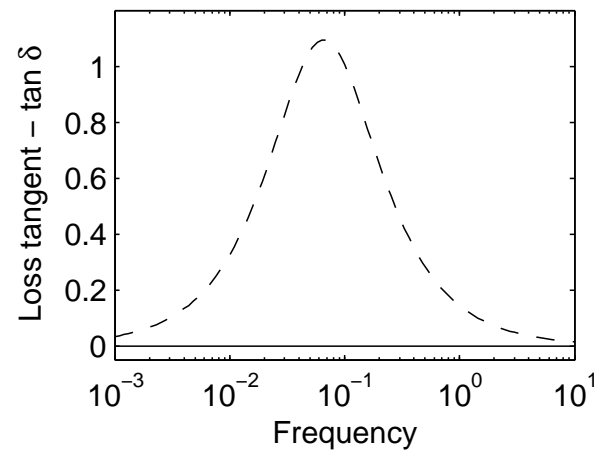

Figure 2: Frequency dependence of the loss tangent, $\tan \delta$. The solid line denotes material 1 while the dashed line denotes material 2.

change and as material 2 exhibits a clear frequency dependence this endpoint moves in accordance with the plots in Fig. 1. For low frequencies the lens region is very narrow. As the frequency is increased the lens region first expands with increasing frequency and then narrows again. In accordance with the previous plots the storage modulus of the composite will increase with frequency and the loss will peak during the transition frequencies.

\section{Theory}

The effective moduli for an elastic composite can be computed by homogenization assuming that a unit cell can represent the periodic material (Bensoussan et al., 1978; Sanchez-Palencia, 1980). For a two-phase periodic heterogeneous material (cf. Fig. 4) having the stiffness tensors $C_{i j k l}^{1}$ and $C_{i j k l}^{2}$ corresponding to the black $\left(\Omega_{1}\right)$ and white $\left(\Omega_{2}\right)$ domains, respectively, the

effective stiffness can be computed, based on the displacements $\chi$ of some test problems, by evaluating the volume integral over the unit cell

$$
C_{i j k l}^{H}=\frac{1}{|\Omega|} \int_{\Omega} C_{p q r s}\left(\varepsilon_{p q}^{0(k l)}-\varepsilon_{p q}\left(\chi^{k l}\right)\right)\left(\varepsilon_{r s}^{0(i j)}-\varepsilon_{r s}\left(\chi^{i j}\right)\right) \mathrm{d} \Omega
$$



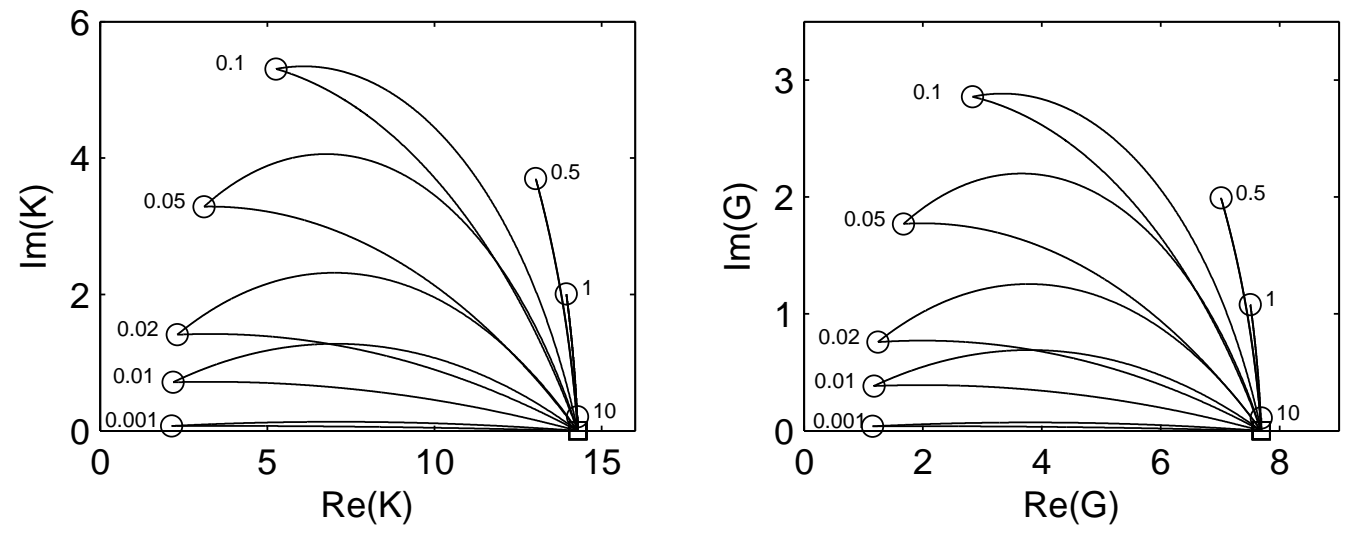

Figure 3: Frequency dependence of the bounds for two-dimensional viscoelastic composites based on the materials described in Table 1. The small numbers indicate the frequency associated with each set of bounds (Gibiansky and Lakes, 1997).
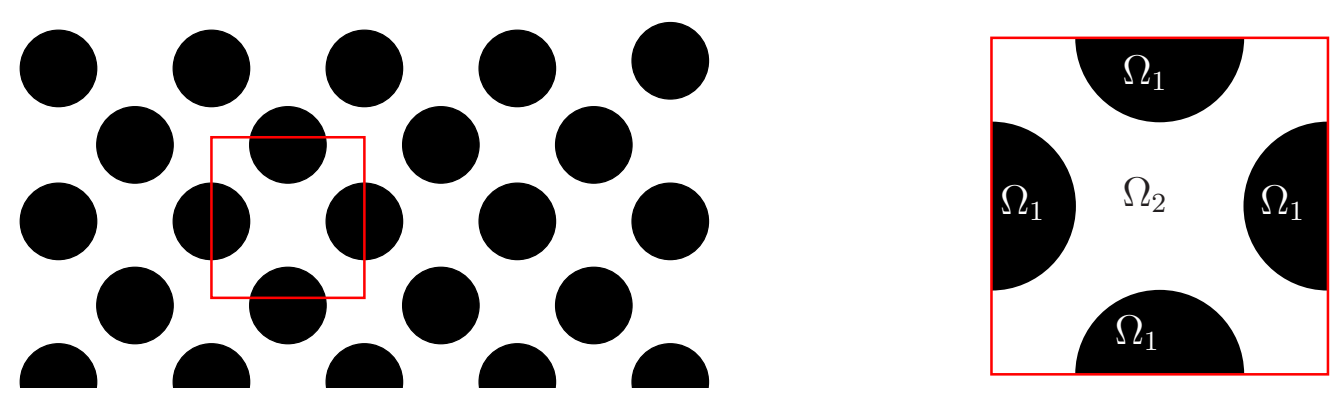

$\Omega$

Figure 4: An illustration of a periodic medium and a representative unit cell. 
where $\varepsilon_{r s}\left(\chi^{i j}\right)=\left(\chi_{r, s}^{i j}+\chi_{s, r}^{i j}\right) / 2$ is the linear strain tensor and $\varepsilon^{0(i j)}$ is a second order tensor with a single non-zero entry at $\varepsilon_{i j}^{0(i j)}$. The local stiffness tensor, $C_{i j k l}$, depends on the distribution of material within the unit cell and is here denoted by the indicator function $\mathcal{I}$

$$
C_{i j k l}= \begin{cases}C_{i j k l}^{1}, & \text { if } \mathcal{I} \in \Omega_{1} \\ C_{i j k l}^{2}, & \text { if } \mathcal{I} \in \Omega_{2}\end{cases}
$$

where each of the stiffness tensors, $q=\{1,2\}$, are defined as

$$
C_{i j k l}^{q}=\frac{E^{q}}{d(1+\nu(1-d))} \delta_{i j} \delta_{k l}+\frac{E^{q}}{1+\nu}\left(\delta_{i k} \delta_{j l}+\delta_{i l} \delta_{j k}-\frac{2}{d} \delta_{i j} \delta_{k l}\right)
$$

The displacement fields $\chi^{k l}$ are obtained as the solutions to a set of cell problems with periodic boundary conditions obeying the elasticity equations (2). The state equations can in weak form be stated as

Find $\chi^{k l} \in V^{3}$ such that

$$
\int_{\Omega} \varepsilon_{i j}(\mathbf{v}) C_{i j p q} \varepsilon_{p q}\left(\chi^{k l}\right) \mathrm{d} \Omega=\int_{\Omega} \varepsilon_{i j}(\mathbf{v}) C_{i j p q} \varepsilon_{p q}^{0(k l)} \mathrm{d} \Omega, \quad \forall \mathbf{v} \in V^{3}
$$

where $\chi^{k l}$ is $V$-periodic and corresponds to a straining of the unit cell in each normal and shear direction. Due to symmetry of the stiffness tensor only three and six cases need to be considered for 2D and 3D, respectively.

In a finite element formulation (14) yields a linear equation system

$$
\mathbf{K} \mathbf{u}^{k}=\mathbf{b}^{k}
$$

where the stiffness matrix and load vectors are given by

$$
\mathbf{K}=\int_{\Omega} \mathbf{B}^{T} \mathbf{D B} \mathrm{d} \Omega, \quad \mathbf{b}^{k}=\int_{\Omega} \mathbf{B}^{T} \mathbf{D B} \mathbf{u}_{0}^{k} \mathrm{~d} \Omega
$$

and periodic boundary conditions are imposed, and where $k$ is the number of load cases (three or six). The strain displacement matrix is denoted B and the constitutive matrix $\mathbf{D}$ while the pre-strain is given in terms of the prescribed displacements in $\mathbf{u}_{0}^{k}$. 


\subsection{Viscoelasticity - The correspondence principle}

The correspondence principle states that it is possible to compute the solution to a viscoelastic problem by the corresponding elastic solution. The viscoelastic solution is obtained by substituting the static stiffness tensor $\mathbf{C}$ with its Laplace transformed counterpart multiplied by the transform variable, i.e. $s \hat{\mathbf{C}}(s)$. For a harmonic excitation this can be simplified to substituting the static stiffness tensor by the Fourier transformed counterpart, the complex stiffness $\mathbf{C}^{*}(\omega)$ (cf. Lakes (2009)). By applying the correspondence principle to the homogenization procedure the effective complex stiffness can be computed in analog to the effective static stiffness using the same formulas and methods except that complex arithmetics are needed. In practice we solve (14) where the stiffness tensor $\mathbf{C}$ is complex.

\subsection{Investigating the bounds}

The bounds by Gibiansky and Lakes (1997) for the material parameters mentioned previously at frequency $\omega=0.01$ are plotted in Fig. 5. The results of a simple parameter study of the effective moduli are also presented as markers in the figure. By homogenizing a $30 \times 30$ element (2D) square unit cell having a square elastic inclusion (material 1) of varying size embedded in a viscoelastic matrix (material 2) the upper bound is approached for the complex bulk modulus. For orthotropic materials with square symmetry two shear moduli exist and as seen from the figure one of these shear moduli violates the isotropic upper bound while the other stays inside the bounds.

For the opposite material configuration, a viscoelastic square embedded in an elastic matrix, it is possible to approach the lower bound for the complex bulk modulus, while the isotropic bounds for the shear modulus are again violated due to the lack of isotropy. Furthermore, it is evident that when the upper bound is approached the left part is well resolved using a coarse unit cell representation, while the right part of the upper bound is difficult to resolve (indicated by the distance between the markers). In order to reach the bound in this region a high resolution is required to resolve fine microstructural details with very thin layers of the lossy material phase. The same observation is done for the upper bound on the shear modulus.

\subsection{Topology optimization}

In the previous section the bounds were reached using a simple orthotropic microstructure with square symmetry. However, obtaining isotropic microstructures by pure intuition is more difficult, thus the use of topology 

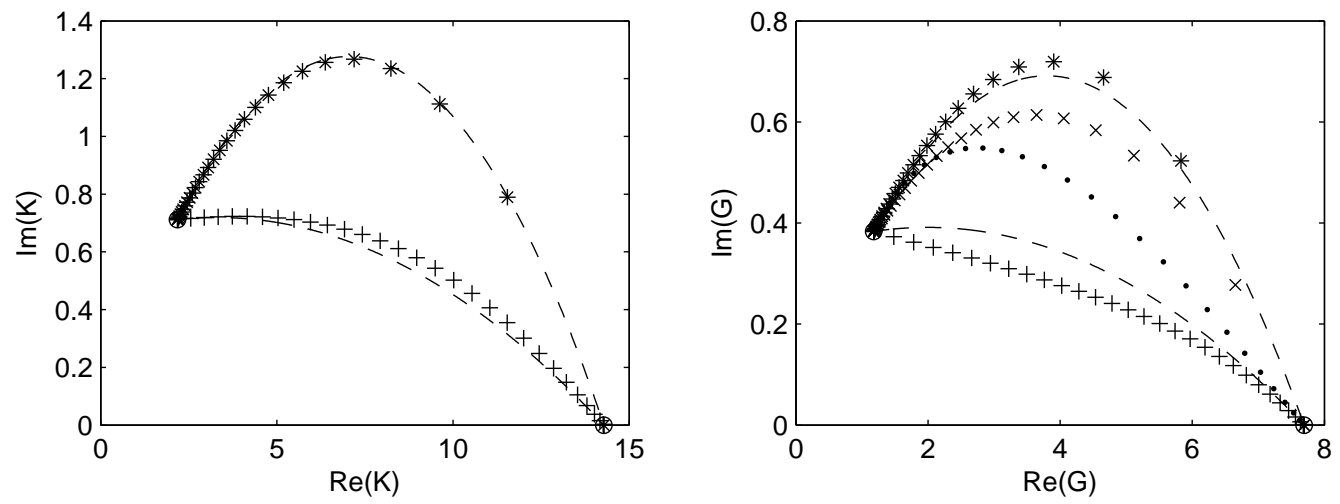

Figure 5: Upper and lower bounds for an isotropic composite consisting of materials mentioned in Table 1. The asterisk $\left(^{*}\right)$ and cross (x) markers correspond to the composite with a square inclusion of elastic material in a viscoelastic matrix. Plus (+) and dot (.) is vice versa. As the microstructure only exhibits square symmetry two shear moduli exist, hence four datasets are plotted in the right figure. Bounds from Gibiansky and Lakes (1997).

optimization is proposed in order to obtain microstructures that approach the bound. Furthermore, an automated process for obtaining microstructures makes it possible to impose multiple constraints such as e.g. volume and/or connectivity constraints.

The discrete problem of (12) is relaxed by introducing a continuous parameter $\rho \in[0 ; 1]$, named the relative density, which indicates the fraction of phase-1 material (the stiff phase) in every material point while the rest is phase- 2 material. As the optimization proceeds, the material points may take any intermediate value, however, towards the end a $0-1$ solution is desired such that the composite can be produced and makes physical sense. Typically, a power-law scheme is chosen for the interpolation

$$
C_{i j k l}(\rho)=\rho^{p} C_{i j k l}^{1}+(1-\rho)^{p} C_{i j k l}^{2}
$$

in which $\rho$ is the relative density of phase 1 while $p$ is a penalization parameter that makes intermediate design variables values uneconomical in the optimization process.

For topology optimization of multi-material problems in the frequency domain it has previously been reported (Sigmund and Jensen, 2003) that using a penalization factor of $p=1$ (linear interpolation) works well. However, 

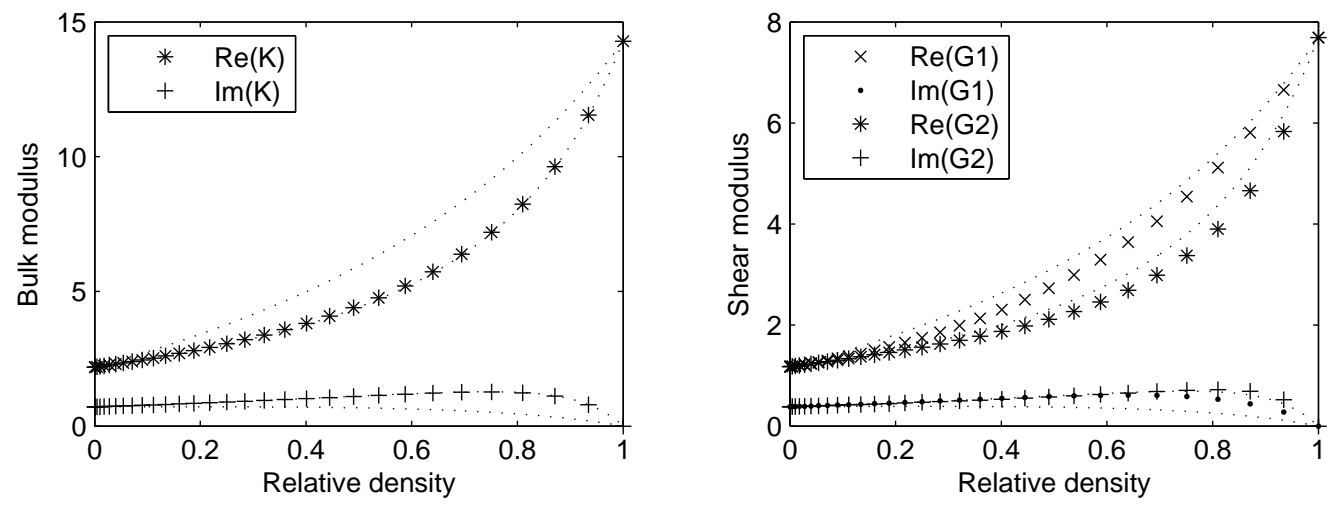

Figure 6: Effective complex moduli vs. relative density corresponding to the composite with a square inclusion of elastic material in a viscoelastic matrix for $\omega=0.01$. Two shear moduli exist as the material exhibits square symmetry. Bounds (Gibiansky and Lakes, 1997) are plotted as dotted lines.

the problem at hand is different and hence we investigate the consequences of choosing another penalization parameter. In order to investigate the influence from the penalization parameter, the effective complex moduli of the structures used in the previous section are plotted versus the relative density in Fig. 6. It is clearly seen that for both the real part of the bulk and shear modulus a power-law interpolation with an exponent larger than one, resulting in a convex function, would be representative. However, for the complex part it is seen that the largest imaginary part is present when the relative density is approximately 0.8 and that it is lower in both ends of the density range. This is approximated much better by a concave function i.e. power-law with exponent less than one. As a compromise no penalization is used, i.e. $p=1$ for both real and complex moduli, which yields a linear interpolation function. As can be seen in Fig. 7, the interpolation violates the lower bound for the complex bulk modulus. However, this is not seen as a problem for an objective function where the imaginary part of the modulus is maximized since material points having intermediate densities perform worse than black/white.

In relation to vibration damping it is of great interest to approach the upper bound for the complex modulus as this, for a given real modulus, will result in the highest possible damping cf. (10). The problem of distributing material within a unit cell in order to achieve maximum damping is formu- 


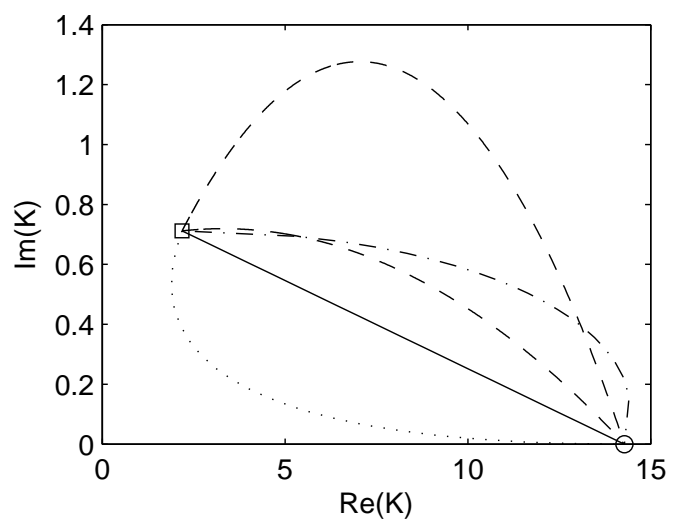

Figure 7: Interpolation function from (16) and bounds (dashed lines) (Gibiansky and Lakes, 1997). Solid line is the interpolation with $p=1$, Upper dash-dotted line is interpolation with $p=0.5$ and the dotted lower line is interpolation $p=2$.

lated as a topology optimization problem:

$$
\begin{aligned}
& \max _{\rho \in \mathbb{R}^{N}} \operatorname{Im}\left(K^{H}\right) \quad \text { Loss modulus } \\
& \text { subject to } \quad \mathbf{K u}^{k}=\mathbf{b}^{k} \quad \text { for } k=1, \ldots, 3(d-1) \quad \text { State equations (15) } \\
& \operatorname{Re}\left(K^{H}\right)<K_{\min } \quad \text { Minimum storage } \\
& g<0 \quad \text { Isotropy constraint } \\
& 0 \leq \rho_{e} \leq 1, \quad \text { for } e=1, \ldots, N \quad \text { Box constraints }
\end{aligned}
$$

where $\mathrm{N}$ denotes the number of design variables and $K_{\min }$ denotes the minimum desired real bulk modulus. As the underlying homogenization method computes the homogenized stiffness matrix the bulk and shear moduli can be computed assuming isotropy based on the $C_{11}^{H}$ and $C_{12}^{H}$ coefficients. However, in order to increase the robustness in case of anisotropic intermediate designs the bulk modulus is computed as the average of two expressions. In $2 \mathrm{D}$ this yield

$$
K^{H}=\left(C_{11}^{H}+C_{22}^{H}+2 C_{12}^{H}\right) / 4
$$

and the shear modulus is computed as

$$
G^{H}=\left(C_{11}^{H}+C_{22}^{H}-2 C_{12}^{H}+2 C_{33}^{H}\right) / 6
$$

The isotropy constraint is formulated as the error between the effective stiffness matrix and the corresponding isotropic stiffness matrix based on the 
homogenized $K^{H}$ and $G^{H}$ i.e.

$$
g=\sum_{i=1}^{6} \sum_{j=1}^{6} \frac{\left(C_{i j}^{H}-C_{i j}^{i s o}\right)^{2}}{\left(C_{11}^{H}\right)^{2}}-\varepsilon^{2}<0
$$

where $\epsilon$ is a small number (i.e. $10^{-3}$ ).

\subsubsection{Connectivity of the elastic phase}

Connectivity of the elastic phase can be ensured in different ways. One option is to impose a stiffness constraint assuming that the viscoelastic phase is softer than the elastic one and thereby force connectivity between elastic material. This, however, is problematic when the frequency goes up as the stiffness of the viscoelastic material will increase and the connectivity of the elastic phase cannot be ensured.

As a better alternative we solve an additional homogenization problem, namely a conductivity problem similar to Sigmund (1999) on top of the existing problem. Using the same design but assigning good conductive properties to the elastic phase and poor to the viscoelastic phase, connectivity can be ensured by enforcing a lower bound on the conductivity i.e. an additional constraint

$$
g=1-\sigma^{H} / \sigma_{0} \leq 0
$$

where $\sigma_{0}$ is the desired minimum effective conductivity.

Cross property bounds between bulk modulus and electrical/thermal conductivity exist for isotropic materials with real material properties and are described in Gibiansky and Torquato (1996). These bounds are also constructed using the translation method and span an area in the conductionbulk modulus plane. The cross property bounds for bulk storage and conductivity can be seen in Fig. 8 where three infeasible property combinations are indicated, namely the combinations $(K=3, \sigma=0.1),(K=3, \sigma=0.2)$, $(K=4, \sigma=0.2)$. These points are marked as they belong to the set of investigated material designs and become unrealizable due to the conductivity constraint and cross property bound.

Alternatively, connectivity can be ensured by constraining the lowest eigenfrequency of the metallic phase in the unit cell to be greater than zero (letting the stiffness of the second phase vanish) (Wang et al., 2011). However, there are two issues with such a procedure for the present design problem. The first is that the computational effort is much larger than solving 


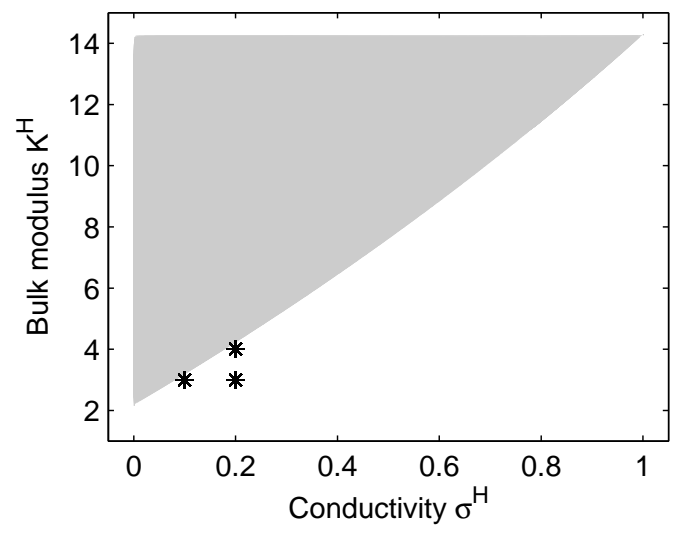

Figure 8: The shaded area is the feasible set of conductivity and bulk storage properties. The three markers indicate the points $(K=3, \sigma=0.1),(K=3, \sigma=0.2)$ and $(K=4$, $\sigma=0.2$ ) which lie outside the cross property bounds (Gibiansky and Torquato, 1996) and thus cannot be realized.

the heat conduction problem. The second, which might be more problematic, is that it is not straightforward to impose boundary conditions for the associated eigenvalue problem as the metallic structure may not be present at the boundary of the domain cf. Fig. 9.

\section{Implementation}

In order to solve the inverse homogenization problem by topology optimization a numerical homogenization procedure (cf. Guedes and Kikuchi (1990)) is implemented. The practical implementation is twofold, one for the two-dimensional problem implemented in Matlab and another for the threedimensional problem implemented using a parallel $\mathrm{C}++$ framework DFEM (Aage and Lazarov, 2013), however, they have much in common. They both use linear elements and the periodic boundary conditions are imposed by sharing nodes between elements on each side of the unit cell. A single node is fully constrained in order to avoid rigid body motion.

The equation system differs from that of the corresponding elastic problem as it includes complex variables and the system matrix is non-Hermitian which means that neither a direct solver based on a Cholesky factorization nor a conjugate gradient method can be used. Instead the 2D implementation uses a direct solver with LU-factorization while the 3D implementation uses a block version of a Jacobi-preconditioned stabilized bi-conjugate gradient 
method (van der Vorst, 1992). For the 2D problems the unit cells have been discretized using square regular bi-linear finite elements which, for the square domains, consist of $100 \times 100$ elements while the rectangular domains consist of $140 \times 80$ elements. For the $3 \mathrm{D}$ problems the cubic unit cells have been discretized using tri-linear elements and the resolution of either $40 \times 40 \times 40$ or $60 \times 60 \times 60$ elements.

The topology optimization procedure is similar to that in Sigmund (1995) used for elastic materials and the optimization problem is solved using the Method of Moving Asymptotes (MMA) kindly provided by Svanberg (1987). The design is regulated using a Heaviside projection filter (Guest et al., 2004; Sigmund, 2007) in order to get black/white designs. By experience the regularization is only needed when the conductivity constraint is imposed, however, for consistency it is applied to all designs.

Material microstructure design problems are prone to local minima related to small details in the microstructure or reentrant structures. A continuation scheme has been imposed in the discreteness measure of the Heaviside projection filter and the isotropy error $(\varepsilon)$ in order to give the optimizer more freedom in the beginning of the optimization process, and avoid getting stuck prematurely in a local minimum.

\section{Results and discussion}

In this section the composites that realize the upper bound for viscoelastic bulk modulus will be presented both in two and three dimensions. This is followed by manufacturable optimized designs in two and three dimensions which is ensured by the conductivity constraint.

\subsection{Extreme bulk modulus structures in 2D}

Optimizing for the bulk loss modulus the upper bound is approached and in order to find structures along the upper bound, restrictions are imposed on the effective bulk storage modulus. Vigdergauz (1994) presented optimal single scale microstructures for elastic materials, which also showed up in Sigmund (2000) where extremal two phase elastic composites were investigated. The microstructures that exhibit the highest bulk modulus either have a honeycomb structure (near circular void inclusions in a triangulas) which requires a rectangular unit cell or an octagonal structure with circular inclusions of two different sizes supporting a square unit cell. For this first case both unit cell types have been investigated for the maximization of the 


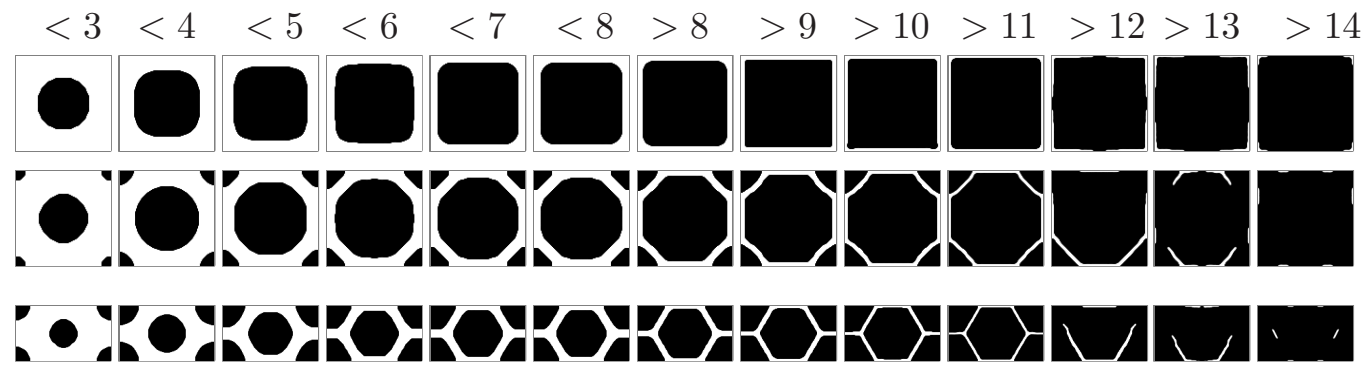

Figure 9: Optimized microstructures for maximum bulk loss modulus. Top row using square design domain and square symmetry, middle row using square design domain with isotropic symmetry and bottom row using a rectangular design domain with isotropic symmetry. Each column corresponds to a different constraint on the effective bulk storage modulus.

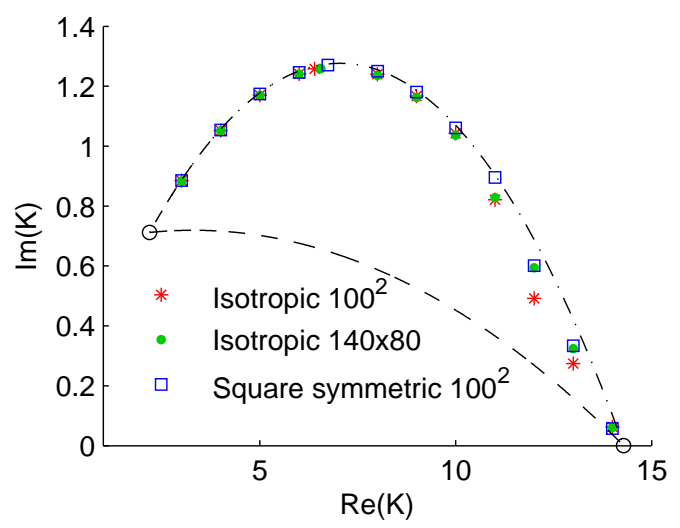

Figure 10: Performance of the optimized microstructures for maximum imaginary bulk modulus along with the corresponding bounds (Gibiansky and Lakes, 1997) for $\omega=0.01$ . The optimized designs are shown in Fig. 9.

bulk loss modulus. The performances of the realized structures are shown in Fig. 10 while the corresponding designs are shown in Fig. 9. From the figure it is clearly seen that Vigdergauz structures are obtained. The upper row exhibits square symmetry while the two lower rows exhibit isotropic symmetry. As the requirements for the bulk storage are increased (moving towards right in the plot) it gets progressively more difficult to resolve the details of the microstructures so, due to the finite mesh resolution, the designs lose geometric symmetry and the performances decrease in comparison to the upper bound. 

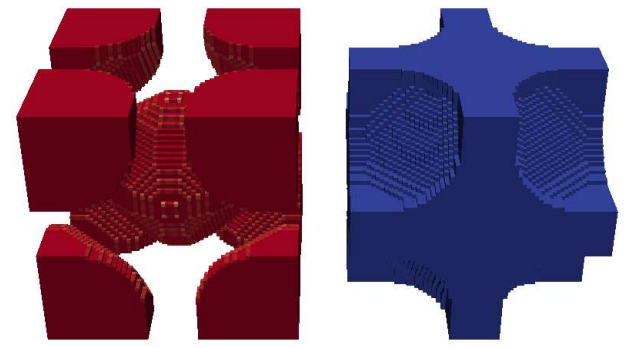

(a)
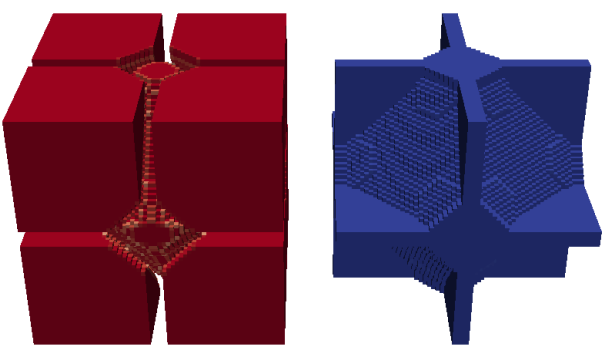

(c)
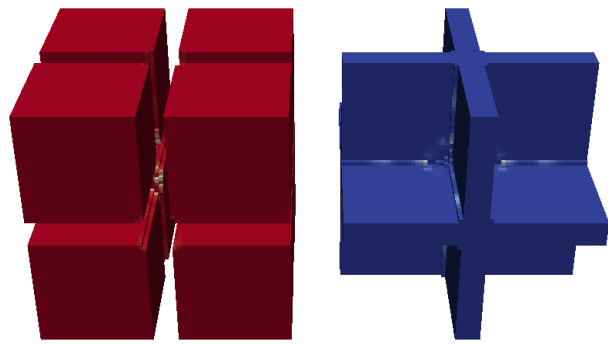

(b)
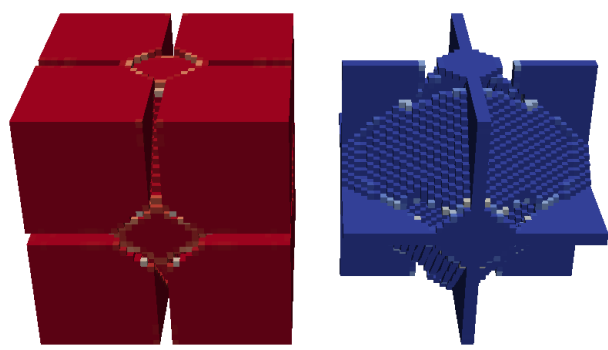

(d)

Figure 11: Optimized microstructures for maximum imaginary part of bulk modulus. Left: Stiff phase. Right: Soft phase. a), c) and d) are isotropic, while b) is cubic symmetric.

\subsection{Extreme bulk modulus structures in 3D}

Figure 11 shows four 3D isotropic microstructures obtained by tracing the upper complex bulk modulus bound. The phase properties used are the same as for the 2D case and the homogenized complex bulk modulus of the obtained designs are compared to the bounds from Gibiansky and Lakes (1993) in Fig. 12, where it can be seen that, as in the 2D case, the right part of the bound is not attained due to the limited discretization. With the coarsest discretization $\left(40^{3}\right.$ elements) the obtained designs deviate more from the bounds, because the loss is maximized by making sure the stiff phase is unconnected. Assuring an unconnected stiff phase requires a larger volume fraction of the soft phase for a coarser discretization, and for the material in Fig. 11(d) there is simply not enough soft material to isolate the stiff phase. From a manufacturing point of view a connected phase is desired in e.g. selective laser sintering. However, the connectivity of the structure should rather be ensured by imposing a constraint than by relying on the coarseness of the discretization. 


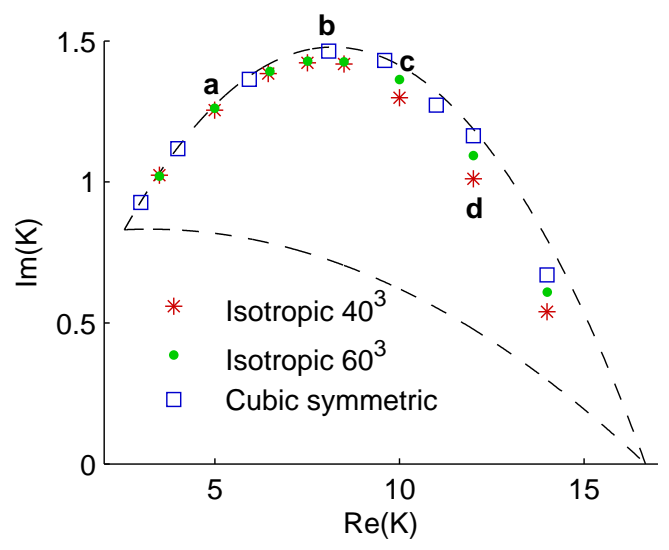

Figure 12: Performance of the optimized three-dimensional microstructures compared to the theoretical bounds (Gibiansky and Lakes, 1993) for $\omega=0.01$. The letters refer to the designs in Fig. 11 .

\subsection{Manufacturability in $2 D$}

The structures optimized in the previous section might not be realized easily as an inclusion of the elastic phase is embedded into a viscoelastic matrix. The targeted manufacturing process is a two step procedure in which the first step is to create a metallic matrix by selective laser sintering and the second step is to infiltrate the matrix with a viscoelastic filler. Hence, the elastic phase should be connected in order to function as a matrix. The infiltration process also requires the structures to be open. However, for 2D designs, both conditions cannot be fulfilled. In this case we consider the open cell requirement to relate to the out-of-plane direction and thus to be fulfilled.

Based on the above considerations the optimization formulation is modified by adding a conductivity constraint. The optimized designs with this constraint are shown in Fig. 13. Rows one and two are obtained imposing a conductivity constraint of $\sigma^{H}>0.1 \sigma_{0}$ and square and isotropic symmetry, respectively. Rows three and four are obtained imposing a conductivity constraint of $\sigma^{H}>0.2 \sigma_{0}$ for square and isotropic symmetry, respectively. Each column corresponds to a specific constraint on the effective bulk storage modulus ranging from $\operatorname{Re}\left(K^{H}\right)<3$ to $\operatorname{Re}\left(K^{H}\right)>14$ in order to assess the entire upper bound. The designs in row one and three are more easily obtained than the other two due to the lower degree of material symmetry. In comparison to the structures in Fig. 9 (upper row) the designs differ by the 
connection such that the elastic material now forms a matrix instead of being inclusions. For the lowest effective bulk storage the designs deviate more and the joint of the matrix takes a flower shape. This, however, might be a local minima rather than a global one. When tightening the conductivity constraint we generally observe that the connectors between the inclusions get wider.

For the isotropic designs in the second and fourth row the structures do show similarities with the ones obtained without conductivity constraint. Except for the connections between the solid inclusions they also, at least until $\operatorname{Re}\left(K^{H}\right)>9$, roughly consist of two different sized inclusions connected by thin pins. For the designs $\operatorname{Re}\left(K^{H}\right)>9$ until $\operatorname{Re}\left(K^{H}\right)>14$ there is a change in topology that performs better than if the previous topology was adapted to the increased storage demands.

The designs $\left(\operatorname{Re}\left(K^{H}\right)>3, \sigma^{H} / \sigma_{0}>0.1\right)$ and $\left(\operatorname{Re}\left(K^{H}\right)>4, \sigma^{H} / \sigma_{0}>\right.$ 0.2 ) do not fulfill the isotropy constraint completely as the optimizer has a very hard time making the design feasible.

The performances corresponding to the designs are shown in Fig. 14 where the different conditions are indicated by different markers. It is seen that the curves for the square and isotropic symmetry almost overlap. Moving towards right they deviate more as the details within the designs combined with the isotropy constraint make it difficult to obtain feasible designs that are as close to the upper bound as the square symmetric ones. Based on these results a new numerical upper bound can be constructed which bounds the structures with a connected stiff elastic phase.

Due to the cross property bounds shown in Fig. 8 it is seen that designs that both restrict the maximum bulk storage and the minimum conductivity might be infeasible. Thus no designs are presented for $\left(\operatorname{Re}\left(K^{H}\right)<3\right.$, $\left.\sigma^{H} / \sigma_{0}>0.1\right),\left(\operatorname{Re}\left(K^{H}\right)<3, \sigma^{H} / \sigma_{0}>0.2\right)$ and $\left(\operatorname{Re}\left(K^{H}\right)<4, \sigma^{H} / \sigma_{0}>0.2\right)$.

\subsection{Manufacturability in 3D}

The performance-effect of the conductivity constraint in $3 \mathrm{D}$ is the same as in 2D; it limits how closely the bounds can be approached. This is illustrated in Fig. 16 where the upper bound is traced with a conductivity constraint of $10 \%\left(\sigma^{H} / \sigma_{0}>0.1\right)$.

Opposed to the two-dimensional designs, the designs in three dimensions allow for both phases to be connected. This is clearly seen for the case with cubic-symmetric material design in Fig. 15(a), where the stiff phase 


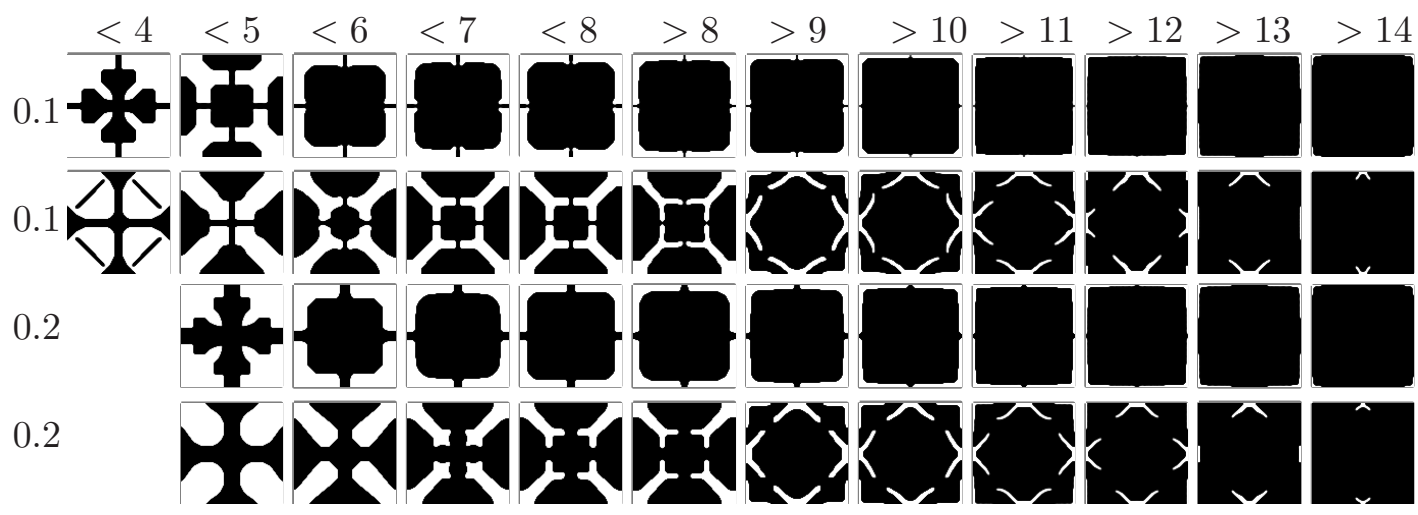

Figure 13: Optimized microstructures for maximum imaginary bulk modulus subject to constraints on storage modulus (columns) and conductivity $(2 \times 2$ rows). The microstructures exhibit square symmetry (rows 1 and 3 ) and isotropic symmetry (rows 2 and 4).

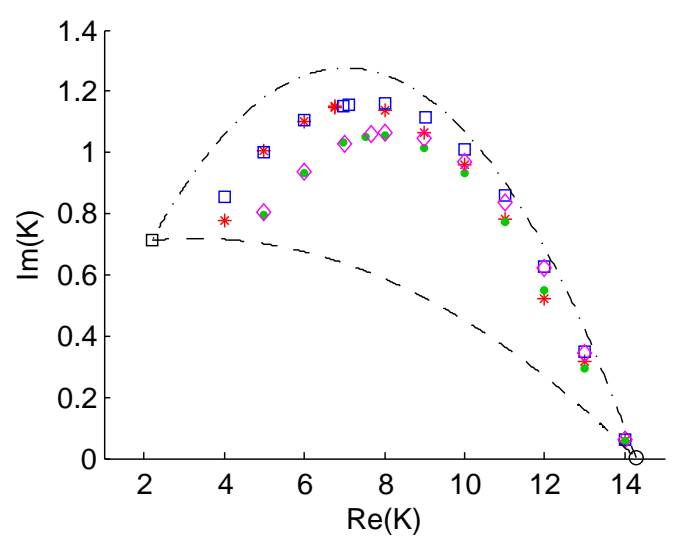

Figure 14: Performance of the optimized microstructures for maximum bulk loss modulus along with the corresponding bounds (Gibiansky and Lakes, 1997) for $\omega=0.01$. The blue square $(\square)$ and red asterisk $(*)$ yields $\sigma^{H} / \sigma_{0}>0.1$ and exhibit square and isotropic symmetry, respectively. The magenta diamond $(\diamond)$ and green $\operatorname{dot}(\cdot)$ yields $\sigma^{H} / \sigma_{0}>0.2$ and exhibit square and isotropic symmetry, respectively. 
is connected with rods compared to Fig. 11(b) where the stiff phase is an inclusion in a lossy matrix.

The constraint ensures some connectivity between the stiff parts, but isolated stiff islands can still appear. This is the case for the isotropic designs in Fig. 15(b) and (c), where the stiff phase is only partially connected and the material is therefore not readily manufacturable. However, as shown in Fig. $15(\mathrm{~d})$ it is also possible to obtain a manufacturable isotropic material using the constraint. The differences in the designs originate from the two different initial guesses being used. The designs in Fig. 15(b) and (c) were obtained with a stiff cross as an initial guess, while the design in Fig. 15(d) was obtained having stiff spheres in the corners in the initial guess. The performance does not seem to be affected negatively by the full connectivity, since the coarse discretization material next to point (d) in Fig. 16 has the same topology as points (b) and (c). In order to investigate the influence from the discritization the result for a single optimization using a higher resolution $\left(80^{3}\right.$ elements) is presented. The optimized structure is similar to the one in Fig. 15(a) and performs equally good. This indicates that the discretization is not a problem for structures with a moderate storage modulus $\operatorname{Re}\left(K^{H}\right)$.

The conductivity constraint can be used to obtain manufacturable designs, but it does not assure manufacturable designs in 3D. It should be mentioned that requiring a larger conductivity is not guaranteed to assure manufacturability, you still risk either isolated stiff or soft islands.

As a larger storage modulus is required the importance of the conductivity constraint diminishes, and the structures at the rightmost part of the upper bound are identical to the ones obtained without the conductivity constraint.

\section{Conclusions}

This paper presents a method to optimize the damping capabilities of viscoelastic composite materials by designing the material microstructure taking the manufacturability of the microstructure into account. The method is demonstrated for material properties corresponding to a single frequency, however, if the composite material is targeted for a broader frequency range the optimization problem may be changed to a min-max type problem to account for multiple frequencies.

It is demonstrated that the design method is capable of attaining the upper bound for the bulk loss modulus when manufacturability is neglected. If manufacturability is considered the upper bound shrinks such that only 

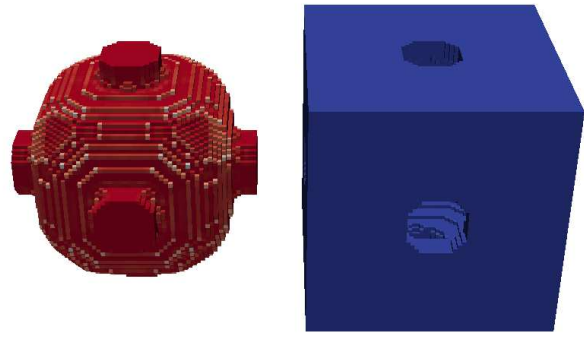

(a)
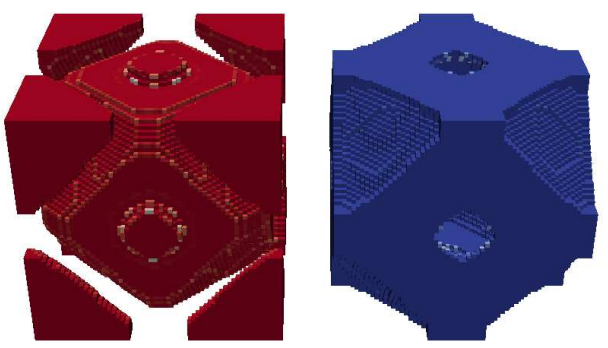

(c)
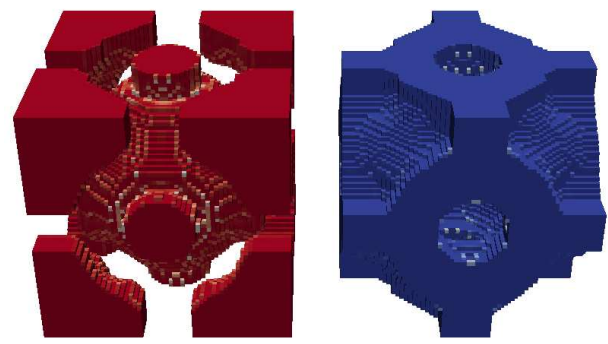

(b)
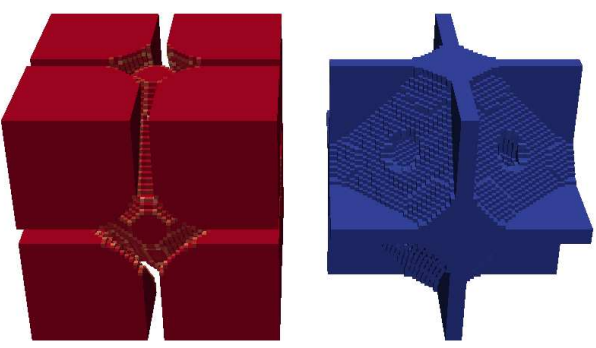

(d)

Figure 15: Optimized microstructures for maximum imaginary part of bulk modulus with a conductivity constraint. In each set of two, left is stiff and right is lossy phase. Design (a) has cubic symmetry and the remaining have isotropic symmetry.

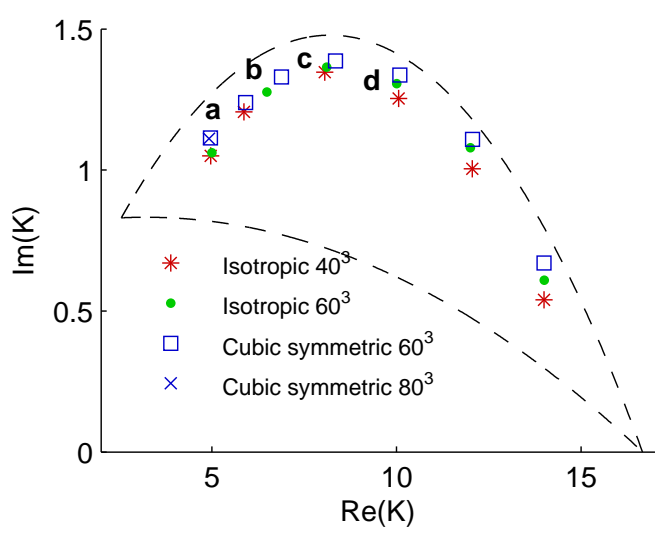

Figure 16: Performance of the microstructures optimized with a conductivity constraint compared to the theoretical bounds (Gibiansky and Lakes, 1993). The conductivity in the stiff phase is required to be at least ten percent of the phase material's conductivity. $\omega=0.01$. The letters refer to the designs in Fig. 15 . 
lower bulk loss modulus and higher bulk storage modulus are obtainable. This provides new numerical bounds for viscoelastic composites which have stiff elastic matrix constructed for example by additive manufacturing.

The quasi-static approach taken in this paper allows for the design of materials for low frequency application where the wavelength is much larger than the size of the unit cell. For applications at higher frequency/shorter wavelength one can resort to a fully dynamic approach by solving several microscale eigenvalue problems based on a Bloch wave expansion. Additionally a multiscale model could be considered which, in addition, would allow the material microstructure to vary throughout the macrostructure.

\section{Acknowledgments}

This research was supported by the Danish Ministry of Science, Technology and Innovation through a Grant to the F.MAT innovation consortium. 


\section{Appendix A. Frequency domain stiffness for Standard Linear Solid}

If a Maxwell element (spring and dashpot in serial) is mounted parallel to a spring the Standard Linear Solid (SLS) model is constructed. The difference from a Maxwell element is that at infinite time, the material has a stiffness, the relaxation modulus.

Both branches in the SLS model is subjected to the same strain and the total stress is the sum of stresses in each branch. The stress-strain relation for the Maxwell element is given by

$$
k \dot{\varepsilon}=\dot{\sigma}_{m}+\frac{1}{\tau} \sigma_{m}
$$

where $\tau=\mu / k$ is the ratio between the viscosity of the damper and the spring stiffness. The stress-strain relation in the spring follows Hooke's law

$$
\sigma_{e}=k_{e} \varepsilon
$$

For the case of harmonic excitation the stress and strain can be represented by exponential functions such that

$$
\sigma(t)=\sigma_{0}^{*} \exp (i \omega t), \quad \text { and } \quad \varepsilon(t)=\varepsilon_{0}^{*} \exp (i \omega t)
$$

Inserting into (A.1) and (A.2) the total stress is given by

$$
\sigma_{t}^{*}=\sigma_{e}^{*}+\sigma_{m}^{*}=\varepsilon_{0}^{*}\left(k_{e}+\frac{i \omega k \tau}{1+i \omega \tau}\right)
$$

which is now independent of time but dependent on frequency. The complex relaxation modulus can be obtained from the relation between stress and strain as

$$
C^{*}(\omega)=\frac{\sigma_{t}^{*}}{\varepsilon_{0}^{*}}=k_{e}+\frac{i \omega k \tau}{1+i \omega \tau}=k_{e}+\frac{k \omega^{2} \tau^{2}}{1+\omega^{2} \tau^{2}}+i \frac{k \omega \tau}{1+\omega^{2} \tau^{2}}
$$

A drawback of the SLS model is that the stiffness drops in two decades of time which compared to real materials is too fast (Lakes, 2009). A more sophisticated model can be obtained by adding more Maxwell elements in parallel. For each extra Maxwell element there will be another 2 parameters, stiffness and viscosity, which will add an extra term to the total stress in (A.4). 
Aage, N., Lazarov, B., 2013. Parallel framework for topology optimization using the method of moving asymptotes. Structural and Multidisciplinary Optimization 47 (4), 493-505.

Bensoussan, A., Lions, J., Papanicolaou, G., 1978. Asymptotic Analysis for Periodic Structures. North-Holland Pub. Co.

Brodt, M., Lakes, R., 1995. Composite materials which exhibit high stiffness and high viscoelastic damping. Journal of Composite Materials 29 (14), 1823-1833.

Cherkaev, A. V., Gibiansky, L. V., 1994. Variational principles for complex conductivity, viscoelasticity, and similar problems in media with complex moduli. Journal of Mathematical Physics 35 (1), 127-145.

Francfort, G. A., Murat, F., 1986. Homogenization and optimal bounds in linear elasticity. Archive for Rational Mechanics and Analysis 94, 307-334.

Gibiansky, L., Lakes, R., 1997. Bounds on the complex bulk and shear moduli of a two-dimensional two-phase viscoelastic composite. Mechanics of Materials 25 (2), $79-95$.

Gibiansky, L., Torquato, S., 1996. Connection between the conductivity and bulk modulus of isotropic composite materials. Proceedings of the Royal Society of London Series A-Mathematical Physical and Engineering Sciences 452 (1945), 253-283.

Gibiansky, L. V., Lakes, R., 1993. Bounds on the complex bulk modulus of a 2-phase viscoelastic composite with arbitrary volume fractions of the components. Mechanics of Materials 16 (3), 317-331.

Gibiansky, L. V., Milton, G. W., 1993. On the effective viscoelastic moduli of two-phase media. i. rigorous bounds on the complex bulk modulus. Proceedings of the Royal Society of London. Series A: Mathematical and Physical Sciences 440 (1908), 163-188.

Guedes, J., Kikuchi, N., 1990. Preprocessing and postprocessing for materials based on the homogenization method with adaptive finite element methods. Computer Methods in Applied Mechanics and Engineering 83 (2), $143-198$. 
Guest, J. K., Prévost, J. H., Belytschko, T., 2004. Achieving minimum length scale in topology optimization using nodal design variables and projection functions. International Journal for Numerical Methods in Engineering 61 (2), 238-254.

Hashin, Z., 1965. Viscoelastic behavior of heterogeneous media. Journal of Applied Mechanics 32 (3), 630-636.

Hashin, Z., 1970. Complex moduli of viscoelastic compositesi. general theory and application to particulate composites. International Journal of Solids and Structures 6 (5), 539-552.

Hashin, Z., Shtrikman, S., 1963. A variational approach to the theory of the elastic behaviour of multiphase materials. Journal of the Mechanics and Physics of Solids 11 (2), 127-140.

Lakes, R. S. and Wineman, A., 2006. On Poisson's Ratio in Linearly Viscoelastic Solids. Journal of Elasticity 85, 45-63.

Lakes, R. S., 2009. Viscoelastic materials. Cambridge University Press.

Lurie, K. A., Cherkaev, A. V., 1986. Exact estimates of the conductivity of a binary mixture of isotropic materials. Proceedings of the Royal Society of Edinburgh, Section: A Mathematics 104 (1-2), 21-38.

Meaud, J., Hulbert, G. M. 2012. Dependence of the dynamic properties of Voigt and Reuss composites on the Poisson's ratio and bulk loss factors of the constituent materials. Journal of Composite Materials, published online, DOI:10.1177/0021998312463456

Millet, P., Schaller, R., Benoit, W., 1981. Characteristic internal friction spectrum of grey cast iron. Journal de Physique Colloques 42 (C5), C5929-C5-934.

Milton, G. W., Berryman, J. G., 1997. On the effective viscoelastic moduli of two-phase media. ii. rigorous bounds on the complex shear modulus in three dimensions. Proceedings of the Royal Society of London. Series A: Mathematical, Physical and Engineering Sciences 453 (1964), 1849-1880.

Otomori, M., Andkjaer, J., Sigmund, O., Izui, K., Nishiwaki, S., 2012. Inverse design of dielectric materials by topology optimization. Progress In Electromagnetics Research 127, 93-120. 
Sanchez-Palencia, E., 1980. Non-homogeneous media and vibration theory. Lecture Notes in Physics, 127. Berlin Heidelberg New York: SpringerVerlag.

Sigmund, O., 1994. Materials with prescribed constitutive parameters: An inverse homogenization problem. International Journal of Solids and Structures 31 (17), 2313-2329.

Sigmund, O., 1995. Tailoring materials with prescribed elastic properties. Mechanics of Materials 20 (4), 351-368.

Sigmund, O., 1999. On the optimality of bone microstructure. In: Pedersen, P., Bendse, M. (Eds.), Synthesis in Bio Solid Mechanics. IUTAM, Kluwer, pp. 221-234.

Sigmund, O., 2000. A new class of extremal composites. Journal of the Mechanics and Physics of Solids 48 (2), $397-428$.

Sigmund, O., Jensen, J. S., 2003. Systematic design of phononic bandgap materials and structures by topology optimization Phil. Trans. R. Soc. Lond. A 361 1001-1019.

Sigmund, O., 2007. Morphology-based black and white filters for topology optimization. Structural and Multidisciplinary Optimization 33 (4-5), 401424.

Svanberg, K., 1987. The method of moving asymptotes - a new method for structural optimization. International Journal for Numerical Methods in Engineering 24 (2), 359-373.

van der Vorst, H., Mar. 1992. Bi-cgstab: A fast and smoothly converging variant of bi-cg for the solution of nonsymmetric linear systems. SIAM J. Sci. and Stat. Comput. 13 (2), 631-644.

Vigdergauz, S. B., 1994. Two-dimensional grained composites of extreme rigidity. Journal of Applied Mechanics 61 (2), 390-394.

Wang, F., Jensen, J. S., Sigmund, O., 2011. Robust topology optimization of photonic crystal waveguides with tailored dispersion properties. J. Opt. Soc. Am. B 28 (3), 387-397. 
Yi, Y.-M., Park, S.-H., Youn, S.-K., 1998. Asymptotic homogenization of viscoelastic composites with periodic microstructures. International Journal of Solids and Structures 35 (17), 2039-2055.

Yi, Y.-M., Park, S.-H., Youn, S.-K., 2000. Design of microstructures of viscoelastic composites for optimal damping characteristics. International Journal of Solids and Structures 37 (35), 4791-4810.

Zener, C., 1948. Elasticity and Anelasticity of Metals. Chicago: University of Chicago Press. 